\title{
LA INFLUENCIA DE LA INVESTIGACIÓN EN LAS POLÍTICAS DE SALUD Y EN LA PRÁCTICA SANITARIA
}

\author{
Andreu Segura Benedicto \\ Institut Universitari de Salut Pública de Catalunya (ISP).
}

\section{INTRODUCCIÓN Y JUSTIFICACIÓN}

Una participación en el último de los encuentros autónomos «MARCELINO PASCUA», que según parece en lo sucesivo formarán parte de las reuniones anuales de la Sociedad Española de Epidemiología (SEE) hace particularmente oportuno el objeto de esta presentación, precisamente porque es propósito principal de la epidemiología el estudio de los determinantes de la salud de las poblaciones, y está claro que, junto a las influencias biológicas, ambientales y sociales que determinan la distribución y el grado de salud y de enfermedad en las poblaciones humanas, los servicios sanitarios son uno de los elementos clave.

Aunque, como pusieron de relieve los salubristas del movimiento higienista del XIX y más modernamente las obras de Dubos ${ }^{1}, \mathrm{Mc}$ Keown ${ }^{2}$ y desde luego Illich ${ }^{3}$, la contribución de los servicios sanitarios, particularmente asistenciales y médicos, durante el siglo pasado fuera mucho menor que la que los médicos reivindicaban como propia.

Las intluencias de Mc Keown y de Illich aparecen en la formulación de los análisis críticos de Dever ${ }^{4}$ y de Lalonde ${ }^{5}$ y tienen mucho que ver en el diseño de políticas como la norteameri-

Correspondencia:

Andreu Segura i Benedicto

Instituto Universitario de Salud Pública de Cataluña Campus de Bellvitage

Universidad Autónoma de Madrid

Carretera de la Feixa Larga, s/n.

08907 Hospitalet de Llobregat. Barcelona cana o la británica y, salvando las distancias, con la estrategia de SALUd PARA TODOS de la OMS.

Pero aunque en general se reconozca que la influencia de los servicios sanitarios sobre la salud es limitada, es evidente también que la asistencia médica, sobre todo, consume una proporción elevada de los recursos económicos de las colectividades, sea a expensas del erario público, mediante fórmulas aseguradoras privadas, o de forma compartida.

Y aun cuando se pueda achacar a los estamentos sanitarios un interés determinado en consumir los más recursos posibles, lo cierto es que el gasto sanitario sigue gozando de gran popularidad, parte de la cual, sin duda, tiene que ver con la respuesta que la sociedad espera del sistema sanitario para aliviar sus problemas de salud.

Pero oportunidad e interés aparte, considerar la investigación sanitaria como un determinante de las intervenciones y en consecuencia como una de las causas de la mejora de la salud de la población, plantea, mutatis mutandis los mismos problemas que se le plantean a la medicina a la hora de evaluar su impacto sobre la salud de la población. Es decir, la atribución de una asociación causal entre los hallazgos de la investigación y su aplicación en la práctica sanitaria.

Aunque el objeto de análisis no permite un diseño del tipo ensayo controlado aleatorio con enmascaramiento doble, porque, logística y coste aparte, no sería pertinente en relación a las preguntas que conviene responder ni tampoco viable, dada la gran cantidad de variables 
que deberíamos controlar para llegar a alguna conclusión operativa.

Por ello me limitaré a formular una serie de consideraciones que tal vez puedan resultar útiles para un futuro análisis sistemático de tal influencia que sabemos que existe y que suponemos que es, en general, positiva. De ahí que me proponga, en primer lugar, caracterizar esquemáticamente el proceso mediante el cual se introducen o se adoptan procedimientos, técnicas e intervenciones relativas a la protección, a la promoción y a la restauración de la salud y ver de reconocer cuál es el papel de la evidencia procurada por la investigación para justificar la adopción de las intervenciones.

En segundo lugar intentaré situar el papel de la investigación en el contexto de la planificación y el diseño de políticas de salud, de manera que se destaque la diversa transcendencia de los distintos enfoques de la investigación: básica y biológica, por un lado y global por el otro, y apuntar el papel integrador que tal vez se podría desarrollar desde la investigación de servicios de salud.

Posteriormente valdrá la pena mencionar, con carácter ilustrativo, algunos ejemplos de investigación potencial o efectivamente influyentes sobre los servicios sanitarios y la salud de la población, sean problemas de salud como el sida, prácticas sanitarias como las ecografías prenatales o la episiotomía rutinaria, o intervenciones sobre factores de riesgo como la hipercolesterolemia.

Finalmente, comentaré algunas ideas relativas a cómo se puede evaluar el impacto de la investigación sobre la salud, más allá de las evaluaciones de productividad basadas en la publicación de los resultados y el factor de impacto de las publicaciones.

\section{EL PROCESO DE ADOPCIÓN DE LAS INTERVENCIONES}

Idealmente podría pensarse que las actividades sanitarias se adoptan como consecuencia de la existencia de evidencia científica suficiente, proporcionada por la investigación biomédica, epidemiológica, etc. En la práctica, sin embargo, sólo sucede así en una pequeña parte de los casos, como es harto conocido.

De hecho, hasta hace muy poco la evidencia empírica más convincente, que es la proporcionada por los estudios experimentales, se ha limitado a un $15 \%$ o un $20 \%$ de las intervenciones y procedimientos ${ }^{7}$. Lo cual contrasta con la general caracterización de la medicina como una ciencia, aun cuando, como he tenido la oportunidad de comentar en otras ocasiones ${ }^{8}$ se trate simplemente de una confusión. La práctica clínica como tal no es una ciencia aunque pretenda legitimarse sobre los conocimientos científicos.

Precisamente la creación de la «Iniciativa Cochrane» que reivindica la medicina basada en la evidencia ${ }^{9}$ (MBE) viene a ser una constatación, indirecta pero concluyente, de este divorcio entre lo que se sabe y lo que se hace. Cabe comentar que los partidarios de la MBE estiman que estas iniciativas han mejorado substantivamente la proporción de las intervenciones que cuentan con pruebas empíricas de su eficacia.

Las razones que explican esta situación son múltiples y complejas pero conviene destacar algunas. Entre ellas, las de carácter histórico. La Medicina es una actividad mucho más antigua que la ciencia, que obedece a necesidades y demandas ancestrales de la población, las cuales persisten a lo largo de los tiempos. Otras, más actuales, tienen que ver con los valores culturales predominantes en nuestras comunidades y hasta con las bases mismas de la organización social, sustentada en el crecimiento económico que exige un consumo cuanto más intenso mejor.

Naturalmente, los intereses sociales y económicos de grupos e instituciones sociales, como la industria sanitaria o las corporaciones profesionales suponen un estímulo constante para la introducción de técnicas, procedimientos e intervenciones, en fases precoces de la contrastación empírica de su utilidad y de su seguridad. Estímulo que favorece y promueve la creación de expectativas de consumo.

Sin embargo, más que aceptar la existencia de intereses de la sociedad en su conjunto hay 
que considerar los conflictos de intereses entre los grupos que la componen, y que los valores que defienden los gobiernos y las instituciones políticas se corresponden con esos intereses, de forma que, a pesar de que formalmente se plantee una política de contención de costes la cual, entre parentesis, sería mucho mejor comprendida y aceptada si se planteara como una política primordialmente de racionalización- realmente se siguen favoreciendo políticas de alto consumo y utilización.

Como ocurre en cualquier situación dialéctica, la solución a este conflicto es inestable y depende de la primacía de unos u otros intereses. Naturalmente, la presión para una racionalización debería venir de los gobiernos, teóricamente preocupados por el bienestar y la seguridad de la población y, prácticamente, por la tendencia al incremento del gasto social entre el que se incluye el sanitario.

Finalmente, hay que tener en cuenta las limitaciones de la evidencia empírica para tomar decisiones, porque no todos los problemas son susceptibles de análisis experimental y porque los estudios experimentales no son suficientes a la hora de garantizar una racionalización de las decisiones sanitarias. Como desideratum podría considerarse que siempre que sea pertinente conviene disponer de evidencia experimental sobre la eficacia de una intervención y que cuando se dispone de ella es necesario incluirla en el proceso de toma de decisiones que debe completarse con otro tipo de consideraciones de caráctcr más político.

\section{UNA LEGITIMACIÓN SANITARIA DE LA INVESTIGACIÓN}

Así las cosas, una primera aportación de la investigación a las políticas de salud y a la práctica sanitaria es, precisamente, la promoción de la neccsidad de racionalización y el establecimiento de criterios y procedimientos que preserven de la utilización de intervenciones sobre las que no exista evidencia suficiente de efectividad, eficiencia, factibilidad y seguridad.

Naturalmente, el problema práctico es cómo definir qué es suficiente evidencia, sobre todo si tenemos en cuenta que, en general, los investigadores y las instituciones de las que dependen están afectados de un sesgo potencial hacia la intervención, porque precisamente sus intereses van en la dirección de aplicar sus descubrimientos.

Nos interesa, pues, determinar hasta qué punto una protointervención sanitaria, una determinada investigación, es la causa de que se mejore o no la salud de la población y cuáles son las consecuencias que produce sobre la organización y la economía de los servicios sanitarios.

El recurso a los métodos desarrollados por la epidemiología más académica para llevar a cabo investigaciones causales - sea desde la perspectiva lógico-deductiva de Aristóteles convenientemente aggiornada por Popper y sus epígonos, o bien desde el empirismo de Bacon que, pasado por Mill y readaptado por Hill, Evans y tantos otros, ha dado como resultado la confección de una suerte de recetas aplicables a las asociaciones observadas entre variables - es impracticable a la hora de analizar la influencia de la investigación sanitaria sobre la salud de forma global, de manera que debemos contentarnos con impresiones generales más o menos agudas y con casos particulares más o menos ilustrativos.

Hay que tener en cuenta, además, que como consecuencia de la escasa importancia que se concede en la práctica a los determinantes extrasanitarios - a pesar del general reconocimiento de la influencia de múltiples políticas sectoriales sobre la salud, como la económica, la educativa, la agrícola, la de comunicaciones, la laboral, etc. - las intervenciones sanitarias se sigan limitando básicamente al ámbito de los servicios médicos. En este sentido, el desarrollo de la epidemiología clínica, de base fundamentalmente biomédica, dificulta la aplicación de los descubrimientos y hallazgos fuera del ámbito estricto de los servicios asistenciales.

Por ello, la evaluación de la utilidad de la investigación se basa en indicadores de producto, como por ejemplo, el número de las patentes registradas, el número de comunicaciones o ponencias presentadas en congresos o el número de artículos publicados y, más aun en el factor impacto de tales publicaciones. 
Aunque se denomine «factor de impacto» este indicador no mide el impacto de los descubrimientos en la práctica sino la consideración que merece a la comunidad profesional, puesto que consiste en las citaciones que recibe un determinado trabajo publicado, cuando se mide bien, o en las que corresponden a la revista donde ha sido publicado, cuando se mide más groseramente.

De todos modos, no hay que olvidar que en el seno de la medicina se vienen desarrollando experiencias críticas y de evaluación suficientemente importantes y probablemente más intensas que en otras profesiones, seguramente porque la medicina requiere más que otras actividades una legitimación científica que es la que permite en última instancia abordar los problemas vitales de los pacientes $y$, en cierta forma, asumirlos, ya sea mediante la expropiación como denunciaba Illich, o compartiéndolos con la comunidad como sería deseable.

\section{ALGUNOS CASOS ILUSTRATIVOS}

Dos tipos distintos de experiencias relacionadas con la investigación y la práctica sanitaria pueden servir de ejemplo. Uno más intersectorial, en la tradición higienista y el otro más circunscrito a las intervenciones asistenciales, más clínico.

En la primera categoría se pueden incluir todas las formalizaciones sobre prioridades de investigación que son consecuencia de la formulación de políticas sanitarias, como la estrategia de salud para todos, que fundamenta la mayor parte de los planes de salud españoles y otras como los Objetivos de Salud que tanto en los Estados Unidos de Norteamérica como en el Reino Unido constituyen las directrices políticas del sector.

En teoría, de estas prioridades y objetivos han de surgir las prioridades en investigación. Algunas han sido elaboradas formalmente, como las que corresponden a la estrategia Salud para $\operatorname{Todos}^{10}$. Otras tienen una vinculación más indirecta y recogen también la influencia de los grupos de intereses, como pueden ser las referidas a los programas $\mathrm{I}+\mathrm{D}$ de la Unión Europea; o al Plan de la Ciencia y, particularmente, a las prioridades específicamente sanitarias formuladas desde el Fondo de Investigaciones Sanitarias.

Ello no obstante, la influencia de una determinada política de priorización, con su reserva presupuestaria correspondiente, no acostumbra a ser suficiente para conseguir sus objetivos, puesto que se requiere una cierta masa crítica de investigadores capacitados e interesados genuinamente en el desarrollo de líneas y de proyectos de investigación que estén en consonancia con las prioridades establecidas.

Digamos que este tipo de iniciativas introduce elementos racionalizadores, en el sentido del realismo ingenuo, en un sistema en el que las motivaciones se generan y se desarrollan de acuerdo con las influencias históricas que componen los paradigmas vigentes de la investigación y con criterios más o menos universales como el prestigio, la utilidad directa, etc.

En ocasiones, además, una cierta visión idealista del investigador provoca que el establecimiento de prioridades de investigación se vea como una intervención paternalista, que condiciona y limita la creatividad. Sin recordar que la disponibilidad de recursos públicos exige de quien tiene la capacidad de distribuirlos una legitimidad suficiente para hacerlo.

Con todo, desde el punto de vista de la eficiencia social, el establecimiento de prioridades debe completarse con una supervisión de los resultados, puesto que la justificación de la financiación no debe depender sólo de la buena intención de los propósitos.

La otra categoria de experiencias se refiere a las intervenciones clínicas, en algunos casos con propósitos preventivos, que teóricamente se justifican mediante los hallazgos de la investigación.

Uno de los ejemplos más ilustrativos es, probablemente, la atención de los pacientes afectados de sida, en la que el desarrollo de fármacos supuestamente eficaces ha influido poderosamente las decisiones terapéuticas, sin respetar muchas veces los requisitos establecidos para la autorización de medicamentos. La 
verdad es que la evidencia disponible sobre la eficacia y la seguridad de muchos de los antiretrovirales sería, si se aplicara a otros problemas de salud, insuficiente para justificar las decisiones de intervención que se han tomado ${ }^{11}$.

Otro ejemplo lo tenemos en la política frente a la hipercolesterolemia ${ }^{12}$. Las recomendaciones para el uso preventivo de fármacos hipolipemiantes se han generalizado mucho antes de disponer de suficientes datos sobre la eficacia de esta intervención. A partir de evidencias convincentes relativas a la erróneamente denominada prevención secundaria, es decir a la prevención de nuevos episodios de cardiopatía isquémica en pacientes coronarios, se ha venido alentando su ampliación a la población general. La publicación del estudio de la pravastatina entre varones escoceses de 45 a 65 años $^{13}$ actuó como el semáforo verde en las carreras de motos. Afortunadamente para los intervencionistas, estudios posteriores parecen confirmar la eficacia de este tipo de prevención. Sin embargo, el impacto de estas intervenciones sobre la población general en la reducción de ataques cardíacos es, en términos absolutos, mucho menos impresionante que si nos fijamos sólo en la disminución del riesgo relativo. Decrementos del orden del 30\% significan si atendemos al NNT (número necesario de tratamientos para evitar un acontecimiento) centenares de personas tratadas durante varios años para prevenir una sola defunción atribuible al colesterol o a la hipertensión.

Podemos referirnos, también, a la evidencia disponible sobre la eficacia y la seguridad de intervenciones diagnósticas o terapéuticas no farmacológicas que se aplican con frecuencia en la asistencia sanitaria. Por ejemplo, al considerar las indicaciones de las ecografías rutinarias en el embarazo ${ }^{14}$, o la práctica de la episiotomía de rutina en la atención obstétrica ${ }^{15}$.

Sin olvidar que la determinación de la eficacia es una condición lógicamente necesaria para justificar la adopción de una intervención, pero no suficiente. En este sentido la distinción entre eficacia y efectividad no es baladí. El célebre ejemplo de Tugwell sobre la diferencia entre la eficacia de un hipotensor estimada en un $80 \%$ a partir de ensayos controlados aleatorios y su impacto real en la población al que se le prescribe, es suficientemente espectacular. Así, según la cobertura real de la población hipertensa, según la capacidad diagnóstica de los clínicos y la pertinencia y adecuación de la prescripción $\mathrm{y}$, también, según el grado de cumplimiento de esta prescripción por parte de los pacientes, nos podemos encontrar con efectividades del orden del $25 \%$.

Un estudio de la AATM de Cataluña (Agencia de Evaluación de Tecnología Médica) en relación con la efectividad de la oxigenoterapia domiciliaria muestra la reducción observada en el impacto de una medida aceptablemente eficaz en condiciones controladas, buena parte de cuyo beneficio se reduce al aplicarla en condiciones reales ${ }^{16}$.

\section{LA VALORACIÓN DEL IMPACTO}

La valoración de la efectividad de la investigación no resulta sencilla, sobre todo si lo que se pretende es disponer de indicadores que nos permitan detectar si una determinada investigación se convierte en una o en un conjunto de intervenciones sanitarias que mejoran efectivamente la salud de la población.

Hemos visto que el recurso a los ensayos controlados aleatorios debe reservarse a la evaluación de la eficacia de intervenciones concretas donde, en general, la innovación o la intervención son la variable independiente que corresponde a la exposición. Otra cosa es el análisis de la efectividad, mucho más cercano al impacto en términos de salud, con el cálculo del correspondiente riesgo atribuible, y finalmente la determinación de la eficiencia, la utilidad o el beneficio, para usar los términos de la jerga de la evaluación económica de los servicios sanitarios.

El empleo de diseños observacionales, particularmente los transversales y los de casos y controles, puede ser un recurso útil. Pero probablemente deban emplearse técnicas de evaluación más cualitativas sin olvidar la investigación histórica, tal vez combinada con estudios de diseño ecológico. 
Como se apuntaba más arriba, la productividad de los investigadores comienza a ser objeto de análisis a partir de indicadores indirectos, digamos de productos intermedios, más que de resultado, como puede ser el número de publicaciones o el factor de impacto de tales publicacioncs. Aspectos ambos que tienen que ver con la bondad o la originalidad de las aportaciones, según los criterios de los comités científicos de las revistas y, en parte con la transcendencia sanitaria, pero sólo en parte.

El impacto sobre la salud de las investigaciones en salud pública depende, como ocurre también en las intervenciones clínicas, de muchos otros factores. Y cuesta trabajo reconocer cuáles son las variables de confusión que deben ser controladas, estandarizadas o estratificadas.

\section{CONCLUSIONES}

Las investigaciones sanitarias de carácter global e intersectorial, propias de la salud pública, son en número, mucho menores que el conjunto de las investigaciones biomédicas y su diseño tropieza, además de con las limitaciones de recursos para llevarlas a cabo, con las dificultades metodológicas consecuentes para contrastar hipótesis generales.

Su transcendencia a la hora de diseñar políticas y de recomendar intervenciones es también limitada, a pesar de la reciente introducción de los objetivos de salud en la elaboración y el establecimiento de prioridades de investigación, debido a que la organización de los scrvicios sanitarios adolece de una orientación biomédica en la que son mucho más fáciles de integrar intervenciones de carácter prescriptivo como las clínicas.

Incluso aquellas intervenciones cuyo propósito explícito es el de la protección o la promoción de la salud resultan más fáciles de poner en práctica en el ámbito de la clínica y las evidencias experimentales que justifican su consideración provienen también de la investigación biomédica, a pesar de que, en general, no cuenten con una contrastación suficiente de su efectividad y su eficiencia. En este sentido, la mayoría de las innovaciones en la prevención de problemas de salud se limita a la prevención secundaria, cuyos efectos indeseables sobre la equidad y la eficiencia no acostumbran a tenerse en cuenta.

La valoración del impacto de la investigación sanitaria probablemente requiera el concurso de planteamientos cualitativos que incluyan la dimensión histórica y que incorporen juicios externos a los del sector. La evaluación por pares es, sin duda, preferible a la ausencia de evaluación pero está sometida a influencias corporativas.

Todo lo cual no es óbice para que, desde la Salud Pública, hagamos un esfuerzo por justificar científicamente nuestras propuestas de intervenciones y, lo que es más importante, introduzcamos elementos de racionalidad a la hora de incorporar en la práctica los resultados de las investigaciones sobre salud, de manera que ello redunde en la mejora de la salud de las poblaciones y las proteja de los riesgos de la precipitación y de los intereses excesivamente parciales.

\section{BIBLIOGRAFÍA}

1. Dubos R. El espejismo de la salud. México: Fondo de Cultura Económica; 1975.

2. Mc Keown T. The role of medicine: dream, mirage or nemesis? Oxford: Basil Blackwell; 1979.

3. Illich I. Némesis médica. Barcelona: Barral editores; 1975.

4. Dever AP. Epidemiology in health services management. Rockville: Aspen; 1984.

5. Lalonde M. A new perspective on the health of Canadians. Otawa: Gov. Printing Office; 1974.

6. Smith D. Where is the wisdom? The poverty of medical evidence. Br Med J 1991; 303: 798-9.

7. Bunker JP. Is efficacy the gold standard for quality assessment?. Inquiry 1988; 25: 51-8.

8. Segura A. La medicina y la ciencia: ¿Coartada o legitimidad? QUARK 1997; 8: 52-9.

9. Sackett DL, Scott W, Rosemberg W, Brian R. Medicina basada en la evidencia. Madrid: Churchill Livingstone; 1997. 
10. OMS. Políticas de investigación para el programa Salud para todos. Madrid: Ministerio de Sanidad y Consumo; 1989.

11. Chalmers TC. The need for early randomization in the development of new drugs for AIDS. J Aquis Immune Syndr 1990; 3: 10-5.

12. Gervas J, Pérez Fernández M. Las hiperlipidemias y la prevención de la cardiopatía isquémica. Med Clín (Barc). 1997; 109:549-52.

13. Shepherd J, Cobbe SM, Ford I, et al. Prevention of coronary heart disease with pravastatin in men with hypercholesterolenia. N Engl J Med 1995; 333: $1301-7$.
14. Ewigman BG, Crane JP, Frigoletto FD, et al. Effect of prenatal ultrasound screening on perinatal outcome. N Engl J Med 1993; 329: 821-7.

15. Argentine Episiotomy Trial Collaborative Group. Routine vs selective episiotomy: a randomised controlled trial. Lancet 1993; 342: 1517-8.

16. Granados A, Escarrabill J, Borrás JM, Sánchez V, Jovell AV. Utilización apropiad y efectividad: la oxigenoterapia crónica domiciliaria en Cataluña. Med Clín (Barc) 1996; 106: 251-3. 